\title{
Transcriptome analysis reveals link between proteasomal and mitochondrial pathways in Parkinson's disease
}

\author{
D. C. Duke1, L. B. Moran ${ }^{1}$, M. E. Kalaitzakis ${ }^{1}$, M. Deprez ${ }^{2}$, D. T. Dexter ${ }^{3}$, R. K. B. Pearce ${ }^{1}$, M. B. Graeber ${ }^{1}$ \\ ${ }^{I}$ Department of Neuropathology, Imperial College London and Hammersmith, Hospitals Trust. Charing Cross Campus, Fulham Palace \\ Road, London W6 8RF, UK \\ ${ }^{2}$ Laboratory of Neuropathology, University Hospital, University of Liège, Liège, Belgium \\ ${ }^{3}$ Department of Cellular and Molecular Neuroscience, Division of Neuroscience and Mental Health, Imperial College London, London, UK
}

\begin{abstract}
There is growing evidence that dysfunction of the mitochondrial respiratory chain and failure of the cellular protein degradation machinery, specifically the ubiquitin-proteasome system, play an important role in the pathogenesis of Parkinson's disease. We now show that the corresponding pathways of these two systems are linked at the transcriptomic level in Parkinsonian substantia nigra. We examined gene expression in medial and lateral substantia nigra (SN) as well as in frontal cortex using whole genome DNA oligonucleotide microarrays. In this study, we use a hypothesis-driven approach in analysing microarray data to describe the expression of mitochondrial and ubiquitin-proteasomal system (UPS) genes in Parkinson's disease (PD). Although a number of genes showed up-regulation, we found an overall decrease in expression affecting the majority of mitochondrial and UPS sequences. The down-regulated genes include genes that encode subunits of complex I and the Parkinson's-disease-linked UCHL1. The observed changes in expression were very similar for both medial and lateral SN and also affected the PD cerebral cortex. As revealed by "gene shaving" clustering analysis, there was a very significant correlation between the transcriptomic profiles of both systems including in control brains. Therefore, the mitochondria and the proteasome form a higher-order gene regulatory network that is severely perturbed in Parkinson's disease. Our quantitative results also suggest that Parkinson's disease is a disease of more than one cell class, i.e. that it goes beyond the catecholaminergic neuron and involves glia as well.
\end{abstract}

Keywords : Alpha-synuclein ; Gene shaving ; Microarrays ; Oxidative stress ; Ubiquitin-proteasome system

\section{Introduction}

Parkinson's disease (PD) is the second most common neurodegenerative disorder after Alzheimer's disease and affects approximately $1 \%$ of the population over 65 years of age [1]. PD is neuropathologically characterised by progressive loss of dopaminergic neurons within the substantia nigra (SN) and neurochemically related systems, the presence of Lewy bodies within surviving nigra neurons [2], and alpha-synuclein deposition in affected brain areas [3] (http://www.ICDNS.org). It is interesting that the pattern of cell death within the substantia nigra is not homogeneous. The lateral ventral tier of the SN (SN1) shows earlier and more severe loss of dopaminergic neurons than the medial dorsal tier of the $\mathrm{SN}(\mathrm{SNm})[4,5]$. The cause of this selective vulnerability is unknown.

Two pieces of the Parkinson's disease puzzle that are repeatedly implicated in the pathological process are mitochondrial complex I inhibition and ubiquitin-proteasomal system (UPS) dysfunction. Gene mutations that cause familial PD have been found in nuclear genes encoding proteins localised to the mitochondria [6-8] and in mitochondrial encoded complex I genes [9, 10]. Mutations of genes encoding components of the UPS, parkin and UCHL1 also have been linked to familial PD [11,12]. There is evidence of dysfunction in both of these systems in sporadic PD. Reduced mitochondrial complex I function has been shown in SN and in muscle cells of patients with sporadic PD [13-15]. Dysfunction of the proteasomal system has been demonstrated in the SN of idiopathic PD patients [16]. Furthermore, mitochondrial complex I inhibitors and proteasome inhibitors can lead to selective loss of dopaminergic neurons in experimental models [17-20]. Therefore, genetic and environmental factors that reduce the function of either of these systems can cause dopaminergic cell loss in the substantia nigra. 
It is now commonly thought that a combination of environmental risk factors along with genetic susceptibility is important for PD aetiology. The key molecular interface where genetics and the environment converge resides at the level of gene expression. Mutations and polymorphisms affect which genes are expressed as do environmental factors that may lead to activation of various transcription factors. Another advantage of looking at gene expression is that the entire human genome has been sequenced so that it is possible to gain a comprehensive picture of gene expression. Therefore, global expression profiling can lead to a much more detailed and complete understanding of complex diseases that have an aetiology that is influenced by genetic and environmental factors, as is the case with sporadic PD. This type of expression profiling can be especially powerful when combined with detailed clinical data.

To date, no other group has published an expression profiling study of the Parkinsonian substantia nigra using a whole-genome microarray set. Our group reported the first complete expression profile of the substantia nigra of well-documented Parkinson's disease cases using a whole-tissue approach [21]. It is increasingly becoming clear that Parkinson's disease is not a disease of one specific cell type (i.e. dopamine neurons) but a much more pervasive disorder that involves glial cells and neurons both within and beyond the nigra. This view is based on evidence from human studies [22-27] as well as from experimental PD models [28-31]. Therefore, it is obvious that, if one limits their experimental design to studying just one cell type, many important aspects of the disease will be missed. We have accordingly chosen to look at gene expression of PD brain tissue, instead of single cell groups, to identify genes involved in the pathogenesis of this disease. Using this approach, Moran et al. [21] identified a number of genes highly deregulated in the PD brain. Among these were several genes encoding mitochondrial and ubiquitin-proteasomal components in the PD substantia nigra.

In the present study, we used a hypothesis-driven approach to determine the extent of the differential expression of mitochondrial and ubiquitin-proteasomal genes in the substantia nigra and cortex of sporadic PD cases. Because both the mitochondria and the UPS appear to play a role in PD pathology, any transcription profile of PD must fully describe the expression of genes related to these two systems. Therefore, in this study, the differential expression of mitochondrial and UPS genes was investigated in detail.

As dopaminergic cell death can be caused by either mitochondrial complex I or proteasomal inhibition, it may be that a subset of our PD cases shows differential expression in proteasomal genes where another subset shows differential expression in mitochondrial genes. Therefore, the patterns of individual subject variability in gene expression were studied using a cluster analysis procedure called gene shaving. Gene shaving clusters genes together based on patterns of a subject's expression variability. These patterns were compared to clinical parameters, integrating expression profiling data with clinical observations.

\section{Materials and methods}

\section{Cases used for study}

Brain tissue from 15 PD cases and 8 non-PD controls was used for this study. Medial as well as lateral substantia nigra and superior frontal gyrus were examined. PD cases and one control brain were provided by the UK Parkinson's Disease Society Tissue Bank at Imperial College London. Tissue from six control brains was obtained from the Laboratory of Neuropathology, University of Liège, University Hospital, Belgium, and one multiple sclerosis (MS) case was provided by the UK Multiple Sclerosis Tissue Bank at Imperial College London. Age at death, onset, disease duration, mode of presentation, medication and presence of dyskinesia were recorded. All PD diagnoses were made in line with international neuropathological consensus criteria (http://www.ICDNS.org). Clinical features (autonomic and cognitive) were graded in a semi-quantitative fashion from 0 to $3+$ (absent to severe) and global scores were established and used to assess differences among PD cases. The average age of death for the PD cases was 80 years (range 68-89 years), the average postmortem delay was $13.4 \mathrm{~h}$ (range 1.3-21.7) and the average tissue $\mathrm{pH}$ was 6.3 (range 6.0-6.7). Seven control cases had no history of a neurological disorder and the disease-specific control case with multiple sclerosis did not show pathology of the substantia nigra. The average age of death for the control cases was 70 years (range 46-81 years), the average post-mortem delay was $12 \mathrm{~h}$ (range 4.0-30.0) and the average tissue $\mathrm{pH}$ was 6.4 (range 6.06.8). There were 47 tissue samples analysed, 15 PD medial nigra, 9 PD lateral nigra, 5 PD superior frontal gyrus, 8 control medial nigra, 7 control lateral nigra and 3 control superior frontal gyrus.

\section{Expression profiling}

Affymetrix HG-U133 A \& B oligonucleotide arrays were used. This array set contains approximately 33,000 well-substantiated human gene sequences. Details of tissue processing, RNA extraction and running of the arrays 
have been given elsewhere [21]. In brief, total RNA was extracted using the RNeasy extraction kit (Qiagen,

Valencia, CA, USA) following the manufacturer's protocol. RNA yields were determined using a spectrophotometer (Gene QuantPro) and the quality of RNA was assessed using agarose gel electrophoresis. Total RNA was used to generate cDNA by reverse transcription using an oligo-dT cDNA synthesis kit (Superscript Choice System, Life Technologies). Biotin-labelled cRNA was prepared from the double-stranded cDNA by in vitro transcription using T7 RNA polymerase and biotin-11-UTP (Enzo, NY, USA). cRNA was purified using an RNeasy clean-up kit (Qiagen, Valencia, CA, USA) and fragmented and hybridised to the arrays. Arrays were then stained with streptavidin phycoerythrin (Molecular Probes), biotinylated antistreptavidin IgG antibody (Molecular Probes) and washed. The arrays were scanned using an Affymetrix Gene Array scanner. All hybridisations met the following quality control conditions: average background $<100 \mathrm{U}$, noise $<5 \mathrm{U}$ and $3^{\prime}: 5^{\prime}$ ratio of actin and GAPDH $<3$. The validation of this microarray data set using Taqman RTPCR has been conducted and some results have been published [21].

\section{Microarray data analysis}

Basic data analysis was conducted as described in [21]. As a superior alternative to CHP/MAS-based normalisation, the GeneChip Robust Multi Array algorithm was used [21]. Statistical analyses were conducted using the Gen-0 software package (http://www.fom.sk.med.ic.ac.uk/Resources/ (E7F8A77A-7675-45BB-B1E51863246FA9A5)/flyer.pdf $>$ ). Schematics of UPS and mitochondrial systems were obtained using GenMAPP version 2 (http://www.Gen MAPP.org >). Details of the data retrieval from Affymetrix GeneChip probe arrays can be found in the Affymetrix Statistical Algorithms Detection Document

(http://www.affymetrix.com/support/technical/whitepapers/sadd_white paper.pdf). After generating mean expression values, Student's $t$ test was used to compare the mean gene expression values of the PD group to those of the control group. The false discovery rate (FDR) [32] criterion $(p<0.05)$ was used to correct for the increased chance of false positives due to multiple comparisons. However, this test was not used as an exclusion criterion. If some transcripts with similar function or in similar pathways passed the FDR and some did not, then both sets of transcripts were reported. This is because the multiple-comparisons test provides a strict yet indiscriminate exclusion criterion. Genes with similar functions that all show a similar expression trend are less likely to be false positives than random individual genes [33]. This approach is becoming increasingly recognised and the strength of looking at microarray data in terms of functional pathways instead of focusing on individual genes is now well documented [33-36].

In the present report, we have chosen to focus on trends in functional gene expression; therefore, we report all genes that have a $p$-value of $p<0.01$ as having differential expression. However, the results of the multiplecomparisons test are reported in all cases. Gene functions were ascertained based on information provided on the Affymetrix website (http://www.affymetrix.com/index.affx>), OMIM (http://www.ncbi.nlm. nih.gov/entrez/query.fcgi?db=OMIM\&itool=toolbar $>$ ) and literature searches using PubMed (http://www.ncbi.nlm.nih.gov/entrez/query.fcgi>).

Examining the clinical and neuropathological features of the different cases in our study, we know that there is within-group variability with respect to disease presentation, clinical symptoms and pathology. Therefore, we expected that there might also be variation in the corresponding gene expression. This individual variance may be important for understanding which genes or molecular pathways are involved in different disease subtypes. We consequently used a clustering procedure, 'gene shaving', which specifically extracts genes with high variability across subjects [37]. Gene shaving extracts statistically significant subsets of genes that show high covariance across individual subjects. For analysis, we built a library of "filters" consisting of gene lists $(\sim 50-$ 200 genes), each coding for specific biological processes or pathways based on annotated transcript information derived from the basic analysis results. To remove spurious variability, we pre-processed the data to extract those probes that were significant in the $t$ test, $p<0.05$. For each of these filters (pathways), one value was calculated for each of the arrays indicating the "pathway expression" as follows: for each transcript, the expression values of the corresponding probes in all the arrays were extracted, creating a data matrix; principal component analysis [38] was then applied to the data matrix to identify the "eigenvector", i.e. the linear combination of the probes that accounts for most of the variability in the data matrix. As an eigenvector is a linear combination of all gene expression values, only the probes that contributed significantly to the eigenvector were of interest and were selected on the basis of the "Gap" test [37]. The linear combination of the probes surviving the test was then used as the expression value of the pathway for each of the arrays. We then investigated the relationship between these clusters and clinical severity ratings as well as the interrelationship between the clusters themselves. 


\section{Results}

\section{Mitochondrial gene expression}

An analysis of nigral gene expression showed both up-and down-regulation of gene expression in PD compared to control tissue. In the medial substantia nigra, there were 26 up-regulated mitochondrial genes, eight of these passing the FDR correction (supplemental Table 1 in "Appendix"). In the lateral substantia nigra, 39 mitochondrial transcripts were up-regulated in the $\mathrm{PD}$, with none passing the multiple comparisons test. Only seven of these were up-regulated in both the SNm and SN1 (supplemental Table 1 in "Appendix"). Genes that were up-regulated transcribed proteins with a variety of functions including mitochondrial electron transport, catecholamine and fatty acid metabolism, and cell death. Supplemental Table 2 in "Appendix" shows the genes that were up-regulated in the SN1 but not in the SNm. There were several transcripts encoding proteins involved in the oxidative stress response, glycine metabolism and proliferation that were only up-regulated in the SN1.

In the cortex, eight mitochondrial genes were up-regulated in PD, these include monoamine oxidase A (MAOA) and dihydrolipoamide branched-chain transacylase that were up-regulated in both SNm and SN1 and A kinase anchor protein 10 that was also up-regulated in the SN1. The expression of solute carrier family 25 member 21 , acetyl-coenzyme A acyltransferase 2, mitochondrial ribosomal protein 63, dimethylglycine dehydrogenase precursor and surfeit 1 was increased only in the cortex. Only five mitochondrial genes were down-regulated in the PD cortex $(p<0.01)$; of these, nipsnap homolog 1 and translocase of inner mitochondrial membrane 10 homolog were also down-regulated in the SNm. The expression of cytochrome b-561, translocase of inner mitochondrial membrane 10 homolog and polymerase (RNA) mitochondrial (DNA directed) (POLRMT) was decreased in the cortex but not in the nigra. POLRMT may be of special interest as it is essential for the initiation of mitochondrial gene transcription [39]. It should be mentioned that the transcripts differentially expressed in the cortex did not pass the multiple-comparisons correction.

The expression of 86 transcripts encoding mitochondrial proteins was down-regulated in the PD SNm, and 38 of those down-regulated passed the FDR correction (supplemental Table 3 in "Appendix"). There were 39 transcripts down-regulated in PD SN1; only four of these passed the FDR correction. Genes showing decreased expression in the PD nigra include proteins that are involved in the mitochondrial electron transport chain, the Krebs/tricarboxylic acid cycle, glutamate metabolism and DNA maintenance as well as inner and outer membrane proteins. Most of the genes down-regulated in the SN1 were also down-regulated in the SNm; however, there were 12 transcripts exhibiting reduced expression in the lateral but not in the medial nigra (supplemental Table 4 in "Appendix").

There were a number of genes involved in the mitochondrial electron transport chain that were differentially regulated in the Parkinsonian substantia nigra including genes that encode proteins of complex I, II and III and the ATP synthase complex (supplemental Fig. 1 in "Appendix"). Of particular interest are nuclear genes that encode subunits of complex I, as reduced complex I function has been implicated in PD pathogenesis [13-15] and mutations of complex I genes in the mitochondrial genome have also been described [10].

\section{UPS gene expression}

The expression of four UPS genes was increased in the PD SNm; SMAD-specific E3 ubiquitin protein ligase 2 (SMURF2), ubiquitin-specific protease 21, ubiquitin-like, containing PHD and RING finger domains 1 (UHRF1) and homocysteine-inducible, endoplasmic reticulum stress-inducible, ubiquitin-like domain member 1 (HERPUD1). Only the stress-induced HERPUD1 passed the multiple-comparisons test and its pathway relationship to the DNAJ family of chaperones is described in greater detail in Moran et al. [21]. Fourteen UPS transcripts were up-regulated in the PD SN1 (supplemental Table 5 in "Appendix") but did not pass the multiplecomparisons test.

Thirty-eight transcripts encoding UPS transcripts were significantly down-regulated in PD SNm, including the PD-linked gene, ubiquitin carboxyl-terminal esterase L1 (UCHL1) (supplemental Table 6 in "Appendix"). Fourteen of these down-regulated genes pass the FDR correction. Twenty-one UPS transcripts were downregulated in the PD SN1. The expression of 11 of these was decreased in the SNm, while ten were only downregulated in the SN1 (supplemental Table 7 in "Appendix"). Numerous species of E2-ubiquitin-conjugating enzymes and deubiquitinating enzymes were down-regulated in the PD nigra, including ubiquitin-specific protease 14, a mutation of which causes an ataxic phenotype in mice [40]. Supplemental Fig. 2 in "Appendix" shows a schematic of genes encoding UPS components that were differentially expressed in PD. 
Three UPS genes were found to be down-regulated in the PD cortex; ubiquitination factor E4B, ubiquitinconjugating enzyme E2M and ubiquitin $\mathrm{C}$, with none passing the multiple-comparisons test. No genes were significantly up-regulated $(p<0.01)$ in the PD cortex.

\section{Gene shaving cluster analysis}

'Gene filters' were obtained by compiling lists of mitochondrial and UPS genes that were significantly different $(p<0.05)$ between PD and control in the SNm. This resulted in four filters: (1) up-regulated mitochondrial genes, (2) up-regulated UPS genes, (3) down-regulated mitochondrial genes and (4) down-regulated UPS genes (for complete gene filters and gene clusters, see supplemental Table 8 in "Appendix"). Gene shaving analysis was also conducted on a gene filter comprised of the dopamine marker genes tyrosine hydroxylase (TH) and dopamine transporter (DAT). Both of these genes are significantly down-regulated in the SN $(p<0.001)$. This analysis was conducted to compare individual expression patterns derived from the above-mentioned filters with this 'dopamine' filter to determine if the pattern seen is the result of a reduction in dopamine neurons within the nigra.

Gene shaving analysis of the 73 mitochondrial transcripts that were up-regulated in PD SNm extracted a cluster that consisted of six probes encoding three genes; thioredoxin interacting protein (TXNIP), monoamine oxidase A and pyruvate dehydrogenase kinase, isoenzyme 4 (PDK4). Supplemental Fig. 3 in "Appendix" shows that there is a clear up-regulation of expression of these genes in the PD cases; however, based on this cluster, four PD cases appear to have expression profiles more like controls. A significantly earlier age at onset $(p=0.01)$ and a significantly longer $(p=0.01)$ duration of disease was found in the PD cases resembling the control group compared to other PD cases.

Gene shaving analysis of the 24 UPS transcripts that were up-regulated in PD SNm reveals a cluster of three probes encoding two genes: SMAD-specific E3 ubiquitin protein ligase 2 and ubiquitin-like, containing PHD and RING finger domains 1. Supplemental Fig. 3 in "Appendix" shows that this analysis reveals a different pattern of individual variation, with half of the PD group having very high expression of this gene cluster and half looking more like the controls. None of the assessed clinical parameters corresponded to this pattern.

Gene shaving analysis of the 167 down-regulated mitochondrial transcripts showed that 143 of them formed a similarly coherent gene cluster (Fig. 1a). All 75 ubiquitin-proteasome transcripts also formed a cluster that varied coherently across subjects (Fig. 1b). Figure 1 shows that there is a definitive overall decrease in expression in the PD groups for both of these clusters of genes and that the pattern of individual variability is similar for the UPS and the mitochondrial transcripts. The pathway expression values produced from the gene shaving analysis are highly correlated $\left(r^{2}=0.0 .935, p<0.0001\right)$. This is also true when the group effect is taken out by looking at the correlation of only the pathway expression values of the PD group $\left(r^{2}=0.804, p<0.0001\right)$ (Fig. 2).

The similarity in expression pattern between the mitochondrial and UPS genes in both controls and PD cases strongly suggests that these systems are highly co-regulated on the transcriptional level also in the normal state. The same analysis was conducted on the SN1 data using an identical set of gene filters. This analysis produced very similar patterns of individual variation of gene expression (Fig. 3) and also revealed a correlation between the patterns of individual expression for down-regulated mitochondrial and UPS gene clusters $\left(r^{2}=0.921\right.$, $p<0.0001)$.

It is interesting to note that when gene shaving was conducted on the cortex data, it demonstrated the same pattern of intra-individual co-correlation as the nigral data. There was general down-regulation of both mitochondrial and UPS gene clusters within the PD group. This shows that there is a trend toward decreased mitochondrial and UPS gene expression in the PD cortex as well as in the PD nigra. There was again a strong correlation between the pathway expression values for mitochondrial and UPS gene clusters derived from the cortex data $\left(r^{2}=0.986, p<0.0001\right)$ (Fig. 4).

Gene shaving analysis of the 'dopamine' filter revealed that both TH and DAT cluster together and there is a general down-regulation of expression seen in the PD SNm compared to control (supplemental Fig. 4 in "Appendix"). However, this individual expression pattern is different from the patterns derived from the mitochondrial and UPS analysis. There is no correlation between the pathway expression values generated from this dopamine cluster with those derived from either the mitochondrial or UPS clusters for the PD cases $\left(r^{2}=0.05\right.$, n.s.; $r^{2}=0.14$, n.s., respectively). This analysis was repeated for the SN1 and similar results were obtained (data not shown). This indicates that the correlation between mitochondrial and UPS pathway expression values is not simply due to a lack of dopamine neuron-related gene expression. 
The correlation of clinical data with microarray results of down-regulated mitochondrial and UPS gene clusters revealed that, for those PD cases with results resembling the control group (PD3, PD6, PD10 and PD15), there was a significantly younger mean age at death $(p=0.02)$.

Fig. 1 The first 15 subjects are PD cases; the following eight are controls. The Y-axis indicates the pathway expression values, which represents the individuals' contribution to gene expression variability within the cluster. a Gene shaving analysis of the down-regulated mitochondrial transcripts showed they formed a similarly coherent gene cluster (cf. supplemental Table 8 in "Appendix"). b All ubiquitin-proteasome transcripts also formed a cluster that varied coherently across subjects (supplemental Table 8 in "Appendix"). There is an overall decrease in expression in the PD SNm for both of these clusters of genes and, furthermore, the pattern of individual variability is similar for the UPS and the mitochondrial transcripts. For both of these clusters PD3, PD6, PD10 and PD15 have higher expression levels of these genes compared to other PD subjects

A

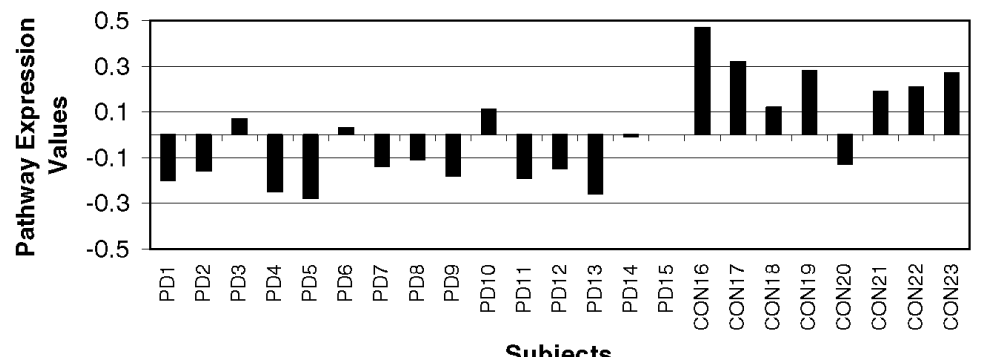

Subjects

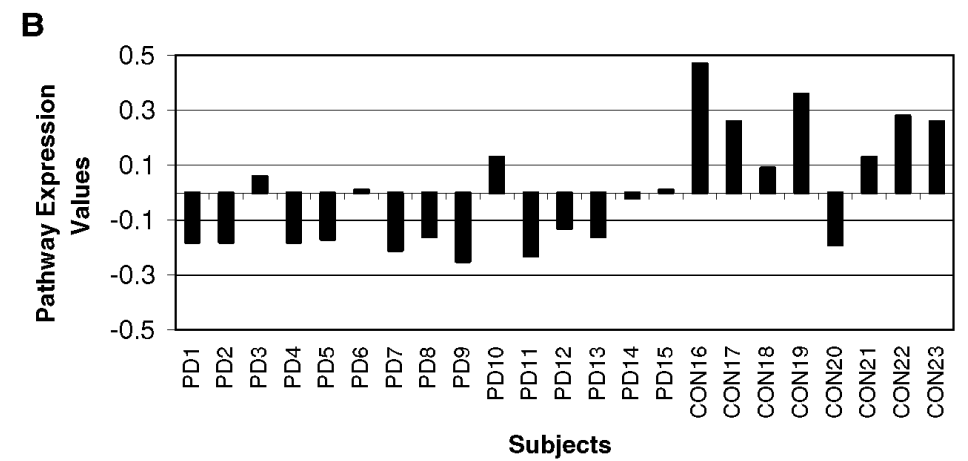

Fig. 2 There is a strong correlation between pathway expression values derived from the gene shaving analysis of down-regulated mitochondrial gene and the pathway expression values derived from the gene shaving analysis of down-regulated UPS genes. Pathway expression values represent the individual subjects' contribution to variability of gene expression within a cluster. Therefore, this correlation indicates that subjects with the lowest expression of mitochondrial genes also had the lowest expression of UPS genes

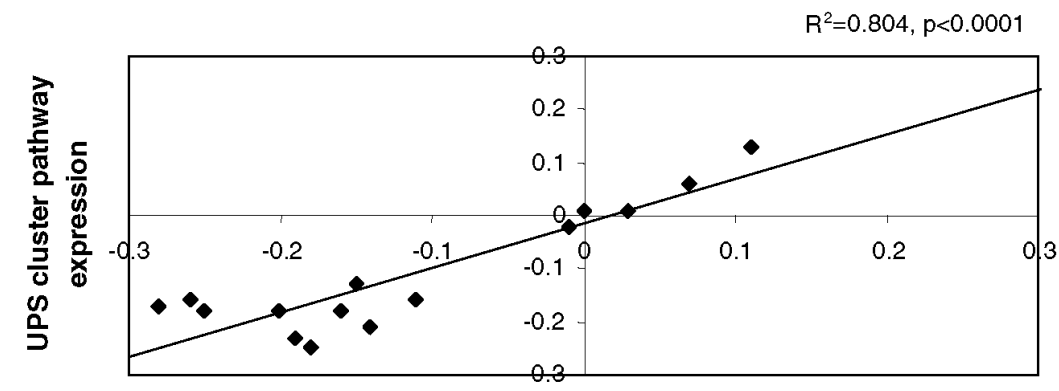

Mitochondrial cluster pathway expression 
Fig. 3 The first nine subjects are PD cases; the last seven represent controls. The $Y$-axis indicates the pathway expression values, which represents the individuals' contribution to gene expression variability within the cluster. a Gene shaving analysis of the down-regulated mitochondrial transcripts using the SN1 gene expression data. $b$ Gene shaving analysis of the down-regulated ubiquitin-proteasome transcripts using the SN1 data. This analysis revealed a similar pattern of individual variability for both gene clusters. As with the SNm clusters, PD3 and PD6 have higher expression levels of these genes compared to other PD subjects. SN1 was not sampled for PD10 and PD15. This shows that the pattern of individual variation seen in the SNm is also seen in the SN1

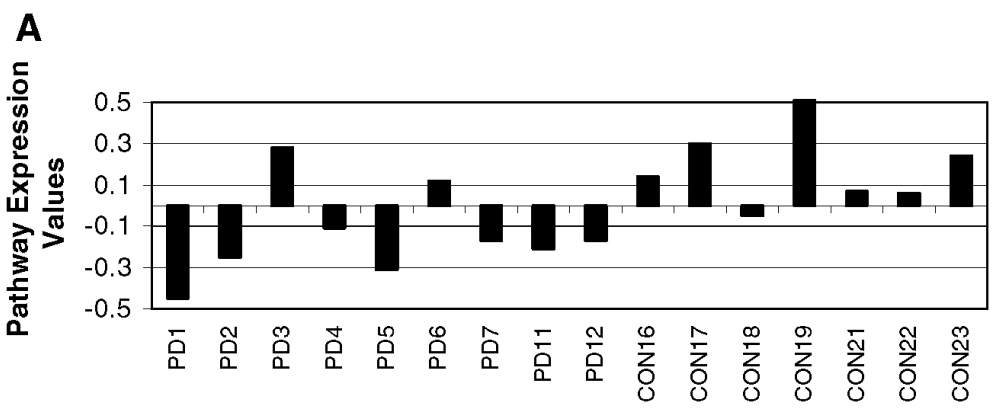

B

Subjects

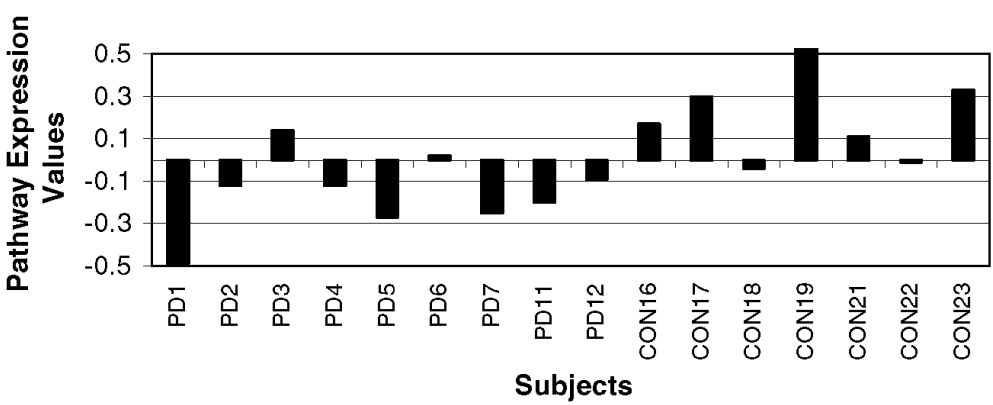

Fig. 4 The first five subjects are PD cases; the following three are controls. The Y-axis indicates the pathway expression values, which represents the individuals' contribution to gene expression variability within the cluster. a Gene shaving analysis of the down-regulated mitochondrial transcripts using the cortical gene expression data. $b$ Gene shaving analysis of the down-regulated ubiquitin-proteasome transcripts using the cortex data. This analysis revealed a similar pattern of individual variability for both gene clusters. For both of these clusters. PD3 and PD15 have higher expression levels of these genes compared to other PD subjects. Cortex was not sampled for PD6 and PD 10. This shows that the pattern of individual variation seen in the SNm and in the SN1 is also seen in the cortex
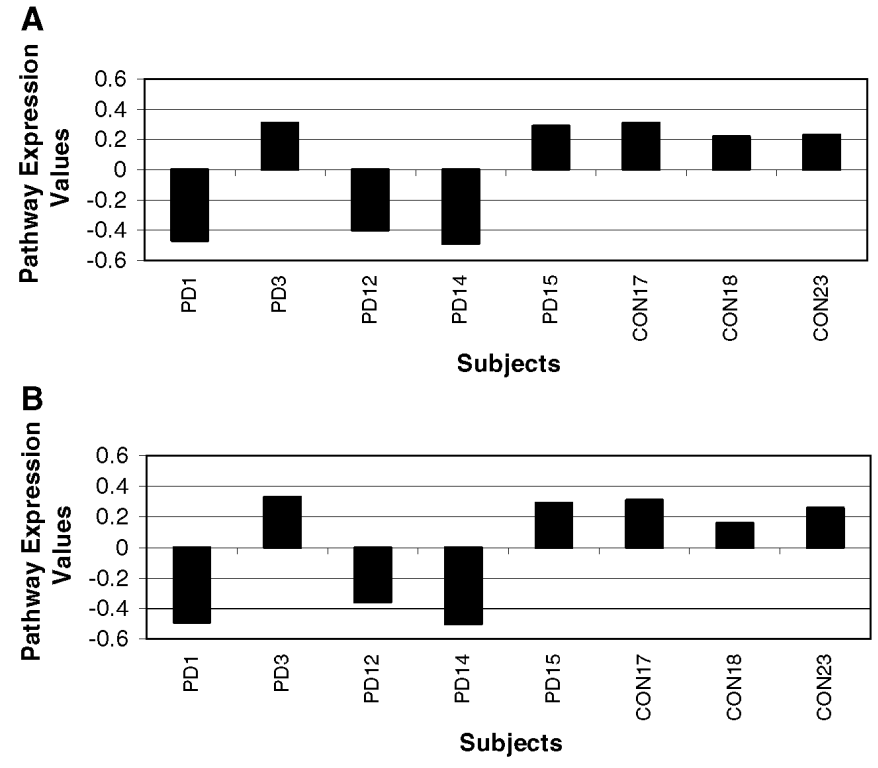


\section{Discussion}

\section{Regulation of mitochondrial and ubiquitin-proteasome gene expression}

Our data demonstrate that there is both up-regulation and down-regulation of transcripts encoding mitochondrial and UPS-related proteins in the PD brain. However, there are many more mitochondrial and UPS transcripts that are down-regulated in the PD nigra than are up-regulated. This is consistent with previous studies that have found reduced mitochondrial [13-15] and UPS [16] activity in the Parkinsonian brain. Our findings are also in line with studies of familial PD [41-43] and work on animal models of PD [44-46], which have independently implicated both metabolic systems in PD. For the first time, it is shown here that the two systems are functionally linked in the human brain.

Of particular interest is the down-regulation of a number of transcripts encoding complex I subunits. Deficits in complex I have been repeatedly implicated in PD pathology [13-15]. There is also a down-regulation of UCHL1, and a loss of function mutation in this gene has been linked to familial PD [6]. Furthermore, there are a number of proteasome subunits that are down-regulated in PD nigra. This is consistent with studies that have shown levels of the PA700 proteasome activator are reduced in the SN in PD [16, 47].

\section{Interrelationship between mitochondria and UPS gene expression}

A particularly important finding in this study is correlation between UPS and mitochondrial gene expression patterns. This is in agreement with recent in vitro as well as animal studies that have provided experimental evidence for the interaction of the ubiquitin-proteasome and mitochondrial systems [19, 48-50]. However, this interdependency between mitochondria and UPS has not been reported in human disease and, furthermore, has not been demonstrated at the level of gene expression. Based on these findings, the functional weakness of one linked system is expected to have a knock-on effect on the other. In turn, therapeutic support for either one of the two components of a higher-order gene regulatory network would be predicted to have a stabilising effect on both. In fact, preventative measures targeted at the human substantia nigra of presymptomatic individuals become an attractive option, and new interesting avenues for effective neuroprotection of the substantia nigra in PD are conceivable.

The evidence obtained in the present study indicating that normal functions of mitochondria and the ubiquitinproteasome system are linked on the transcriptome level deserves special consideration. A very significant correlation between the gene expression patterns of both systems was found using gene shaving cluster analysis. In addition, gene expression of mitochondrial and UPS genes was co-regulated irrespective of PD subgroup, showing that these systems are functionally linked both in disease and in the normal state. Importantly, dysfunction of this higher-order pathway likely resides at the core of the pathological process underlying common sporadic PD. This is in agreement with recent in vitro as well as Parkinson's disease animal model studies that have provided experimental evidence for the interaction of the ubiquitin-proteasome and mitochondrial systems [19, 48-50]. Finding a similar relationship between mitochondria and UPS in welldocumented Parkinson's disease cases strongly supports the validity of these model systems. While the data from the present study suggest that this interaction may be at the level of gene expression, we cannot completely rule out the possibility that the biochemical link between the mitochondria and UPS may be indirect and that factors such as oxidative stress or alpha-synuclein aggregation itself may interfere with the normal function of one or both of these systems [51, 52].

\section{Patterns of mitochondria and UPS gene expression in the PD cortex}

Another important finding of this study is that, using gene shaving analysis, the cerebral cortex was found to show a similar pattern of individual variation in gene expression as the SN. Therefore, the transcriptional changes reported here are not exclusive to the substantia nigra. This strongly suggests that PD is "systemic", at least at the organ level, rather than representing a disease of the substantia nigra. This is supported by a very recent study that found evidence for impaired mitochondrial function in PD visual cortex [22]. The fact that there were fewer cortical transcripts that reached statistical significance is likely due to the small number of cortical samples in this study. Our results suggest that a more comprehensive study describing gene expression in brain areas that are not traditionally associated with PD, like cerebral cortex, may lead to a better understanding of this disease. This view is in keeping with recent histological studies that have described pathological deposition of alpha-synuclein in PD in many brain regions, including frontal cortex [27, 53] (see http://www.ICDNS.org). 


\section{Integration of clinical observation with gene expression data}

Clinical records are often not available for tissue used in human post-mortem disease studies. We are fortunate to have access to not only high-quality tissue but also clinical histories accompanying this tissue. Therefore, our laboratory is in a position to integrate these two aspects of the disease. Using gene shaving analysis, we found clusters of genes that showed similar variability across our subjects. These patterns of individual variability were then compared to clinical parameters. It is interesting that a cluster of three mitochondrial genes that were upregulated in our data set, TXNIP, MAOA and PDK4, characterised four PD cases that had expression values more like controls and also had a significantly younger age of onset. The main risk factor for sporadic Parkinsonism is increasing age and, thus, it is of great interest that a group of our PD cohort with a younger age at onset had a different gene expression profile. At one end of the clinical spectrum, one sees young or even juvenile onset familial cases with identified genetic aetiologies, while with advancing age, one encounters "senile" Parkinsonism and incidental Lewy body disease, present in up to a third or half of those living into their tenth decade and which much more likely reflect the epiphenomena of aging in the human brain. In the middle stand those 'classical' cases, as described by James Parkinson himself, with onset typically in the fifties or sixties, likely owing to a combination of genetic and environmental factors. In the present study, we show that one can find patterns of gene expression that can correspond to clinical parameters. This methodological approach may lead to a greater understanding of disease subtypes and variation in the clinical presentation of PD.

In conclusion, the transcriptomic data reported here strongly support previous protein-based studies that have independently implicated both mitochondrial and UPS dysfunction in PD. In addition, we describe what very likely represents a crucial gene regulatory pathway link between mitochondrial and UPS gene expression in PD. Therefore, any hypothesis addressing the pathobiochemistry of PD has to incorporate both of these systems and their interrelationship.

\section{Acknowledgements}

We would like to thank Dr. Federico Turkheimer for writing the GEN-O analysis program and for guiding us in performing the statistical analysis. We are grateful to the brain donors and their families for providing brain tissue for research. This work is funded through a programme grant from the UK Parkinson's Disease Society Tissue Bank at Imperial College London, funded by the Parkinson's Disease Society of the UK. registered charity, 948776. We would also like to thank the UK Multiple Sclerosis Tissue Bank at Imperial College London. The use of an Affymetrix node in the Department of Neuropathology, funded by the Corsellis Collection, is gratefully acknowledged.

\section{References}

1. Teravainen H, Forgach L, Hietanen M, Schulzer M, Schoenberg B, Calne DB (1986) The age of onset of Parkinson's disease: etiological implications. Can J Neurol Sci 13:317-319

2. Forno LS (1986) Lewy bodies. N Engl J Med 314:122

3. Graeber MB, Dexter D, Pearce RK, Reynolds R (2003) Parkinson's disease: an update. Neuropathol Appl Neurobiol 29:514-515

4. Gibb WR, Fearnley JM, Lees AJ (1990) The anatomy and pigmentation of the human substantia nigra in relation to selective neuronal vulnerability. Adv Neurol 53:31-34

5. Gibb WR (1991) Neuropathology of the substantia nigra. Eur Neurol 31(Suppl 1):48-59

6. Bonifati V, Rizzu P, van Baren MJ, Schaap O, Breedveld GJ, Krieger E, Dekker MC, Squitieri F, Ibanez P, Joosse M, van Dongen JW, Vanacore N, van Swieten JC, Brice A, Meco G, van Duijn CM, Oostra BA, Heutink P (2003) Mutations in the DJ-1 gene associated with autosomal recessive early-onset parkinsonism. Science 299:256-259

7. Zhang L, Shimoji M, Thomas B, Moore DJ, Yu SW, Marupudi NI, Torp R, Torgner IA, Ottersen OP, Dawson TM, Dawson VL (2005) Mitochondrial localization of the Parkinson's disease related protein DJ-1: implications for pathogenesis. Hum Mol Genet 14:2063-2073

8. Valente EM, Abou-Sleiman PM, Caputo V, Muqit MM, Harvey K, Gispert S, Ali Z, Del Turco D, Bentivoglio AR, Healy DG, Albanese A, Nussbaum R, Gonzalez-Maldonado R, Deller T, Salvi S, Cortelli P, Gilks WP, Latchman DS, Harvey RJ, Dallapiccola B, Auburger G, Wood NW (2004) Hereditary early-onset Parkinson's disease caused by mutations in PINK1. Science 304:1158-1160 
Published in : Neurogenetics (2006), vol. 7, pp. 139-148

Status : Postprint (Author's version)

tRNA genes in histologically confirmed Parkinson disease. Neurogenetics 2:121-127

10. Kosel S, Grasbon-Frodl EM, Mautsch U, Egensperger R, von Eitzen U, Frishman D, Hofmann S, Gerbitz KD, Mehraein P, Graeber MB (1998) Novel mutations of mitochondrial complex I in pathologically proven Parkinson disease. Neurogenetics 1:197-204

11. Kitada T, Asakawa S, Hattori N, Matsumine H, Yamamura Y Minoshima S, Yokochi M, Mizuno Y, Shimizu N (1998) Mutations in the parkin gene cause autosomal recessive juvenile parkinsonism. Nature 392:605-608

12. Leroy E, Boyer R, Auburger G, Leube B, Ulm G, Mezey E, Harta G, Brownstein MJ, Jonnalagada S, Chernova T, Dehejia A, Lavedan C, Gasser T, Steinbach PJ, Wilkinson KD, Polymeropoulos MH (1998) The ubiquitin pathway in Parkinson's disease. Nature 395:451-452

13. Schapira AH, Cooper JM, Dexter D, Jenner P, Clark JB, Marsden CD (1989) Mitochondrial complex I deficiency in Parkinson's disease. Lancet $1: 126914$

14. Mizuno Y, Yoshino H, Ikebe S, Hattori N, Kobayashi T. Shimoda-Matsubayashi S, Matsumine H, Kondo T (1998) Mitochondrial dysfunction in Parkinson's disease. Ann Neurol 44:S99-S109

15. Janetzky B, Hauck S, Youdim MB, Riederer P, Jellinger K, Pantucek F, Zochling R, Boissl KW, Reichmann H (1994) Unaltered aconitase activity, but decreased complex I activity in substantia nigra pars compacta of patients with Parkinson's disease. Neurosci Lett 169:126-128

16. McNaught KS, Belizaire R, Isacson O, Jenner P, Olanow CW (2003) Altered proteasomal function in sporadic Parkinson's disease. Exp Neurol 179:38-46

17. Kolata G (1983) Monkey model of Parkinson's disease. Science 220:705

18. Betarbet R, Sherer TB, MacKenzie G, Garcia-Osuna M, Panov AV, Greenamyre JT (2000) Chronic systemic pesticide exposure reproduces features of Parkinson's disease. Nat Neurosci 3:1301-1306

19. Rideout HJ, Larsen KE, Sulzer D, Stefanis L (2001 ) Proteasomal inhibition leads to formation of ubiquitin/alpha-synucleinimmunoreactive inclusions in PC12 cells. J Neurochem 78:899-908

20. McNaught KS, Bjorklund LM, Belizaire R, Isacson O, Jenner P, Olanow CW (2002) Proteasome inhibition causes nigral degeneration with inclusion bodies in rats. Neuroreport 13:1437-144

21. Moran LB, Duke DC, Deprez M, Dexter DT, Pearce RK, Graeber MB (2006) Whole genome expression profiling of the medial and lateral substantia nigra in Parkinson's disease. Neurogenetics (In press)

22. Rango M, Bonifati C, Bresolin N (2006) Parkinson's disease and brain mitochondrial dysfunction: a functional phosphorus magnetic resonance spectroscopy study. J Cereb Blood Flow Metab 26:283-290

23. Orr CF, Rowe DB, Mizuno Y, Mori H, Halliday GM (2005) A possible role for humoral immunity in the pathogenesis of Parkinson's disease. Brain 128:2665-2674

24. Ouchi Y, Yoshikawa E, Sekine Y, Futatsubashi M, Kanno T, Ogusu T, Torizuka T (2005) Microglial activation and dopamine terminal loss in early Parkinson's disease. Ann Neurol 57:168-175

25. van Muiswinkel FL, de Vos RA, Bol JG, Andringa G, Jansen Steur EN, Ross D, Siegel D, Drukarch B (2004) Expression of $\mathrm{NAD}(\mathrm{P}) \mathrm{H}$ :quinone oxidoreductase in the normal and Parkinsonian substantia nigra. Neurobiol Aging 25:1253-1262

26. Wakabayashi K, Hayashi S, Yoshimoto M, Kudo H, Takahashi H (2000) NACP/alphasynucleinpositive filamentous inclusions in astrocytes and oligodendrocytes of Parkinson's disease brains. Acta Neuropathol (Berl) 99:14-20

27. Wakabayashi K, Mori F, Oyama Y, Kurihara A, Kamada M, Yoshimoto M, Takahashi H (2003) Lewy bodies in Betz cells of the motor cortex in a patient with Parkinson's disease. Acta Neuropathologica 105:189-192

28. Smeyne M, Jiao Y, Shepherd KR, Smeyne RJ (2005) Glia cell number modulates sensitivity to MPTP in mice. Glia 52:144-152

29. Marchetti B, Serra PA, L'Episcopo F, Tirolo C, Caniglia S, Testa N, Cioni S, Gennuso F, Rocchitta G, Desole MS, Mazzarino MC, Miele E, Morale MC (2005) Hormones are key actors in gene x environment interactions programming the vulnerability to Parkinson's disease: glia as a common final pathway. Ann N Y Acad Sci 1057:296-318

30. Morale MC, Serra PA, L'Episcopo F, Tirolo C, Caniglia S, Testa N, Gennuso F, Giaquinta G, Rocchitta G, Desole MS, Miele E, Marchetti B (2006) Estrogen, neuroinflammation and neuroprotection in Parkinson's disease: glia dictates resistance versus vulnerability to neurodegeneration. Neuroscience (In press)

31. Zhang W, Wang T, Pei Z, Miller DS, Wu X, Block ML, Wilson B, Zhou Y, Hong JS, Zhang J (2005) Aggregated alpha-synuclein 
Published in : Neurogenetics (2006), vol. 7, pp. 139-148

Status : Postprint (Author's version)

activates microglia: a process leading to disease progression in Parkinson's disease. FASEB J 19:533-542

32. Benjamini Y, Hochberg Y (2000) On the adaptive control of the false discovery fate in multiple testing with independent statistics. J Educ Behav Stat 25:60-83

33. Blalock EM, Chen KC, Stromberg AJ, Norris CM, Kadish I, Kraner SD, Porter NM, Landfield PW (2005) Harnessing the power of gene microarrays for the study of brain aging and Alzheimer's disease: statistical reliability and functional correlation. Ageing Res Rev 4:481-512

34. Blalock EM, Geddes JW, Chen KC, Porter NM, Markesbery WR, Landfield PW (2004) Incipient Alzheimer's disease: microarray correlation analyses reveal major transcriptional and tumor suppressor responses. Proc Natl Acad Sci USA 101:2173-2178

35. Mimics K, Middleton FA, Lewis DA, Levitt P (2001) The human genome: gene expression profiling and schizophrenia. Am JPsychiatr $158: 1384$

36. Quackenbush J (2003) Genomics. Microarrays_-guilt by association. Science 302:240-241

37. Hastie T, Tibshirani R, Eisen MB, Alizadeh A, Levy R, Staudt L, Chan WC, Botstein D, Brown P (2000) 'Gene shaving' as a method for identifying distinct sets of genes with similar expression patterns. Genome Biol 1 :RESEARCH0003

38. Alter O, Brown PO, Botstein D (2000) Singular value decomposition for genome-wide expression data processing and modeling. Proc Natl Acad Sci USA 97:10101-10106

39. Gaspari M, Falkenberg M, Larsson NG, Gustafsson CM (2004) The mitochondrial RNA polymerase contributes critically to promoter specificity in mammalian cells. EMBO J 23:4606-4614

40. Wilson SM, Bhattacharyya B, Rachel RA, Coppola V. Tessarollo L, Householder DB, Fletcher CF, Miller RJ, Copeland NG, Jenkins NA (2002) Synaptic defects in ataxia mice result from a mutation in Uspl4, encoding a ubiquitin-specific protease. Nat Genet 32:420-425

41. Hardy J, Cookson MR, Singleton A (2003) Genes and parkinsonism. Lancet Neurol 2:221-228

42. Hattori N, Kobayashi H, Sasaki-Hatano Y, Sato K, Mizuno Y (2003) Familial Parkinson's disease: a hint to elucidate the mechanisms of nigral degeneration. J Neurol 250(Suppl 3): III2-III10

43. Lim KL, Dawson VL, Dawson TM (2003) The cast of molecular characters in Parkinson's disease: felons, conspirators, and suspects. Ann N Y Acad Sci 991:80-92

44. Betarbet R, Sherer TB, Greenamyre JT (2002) Animal models of Parkinson's disease. Bioessays 24:308-318

45. McNaught KS, Perl DP, Brownell AL, Olanow CW (2004) Systemic exposure to proteasome inhibitors causes a progressive model of Parkinson's disease. Ann Neurol 56:149-162

46. Maries E, Dass B, Collier TJ, Kordower JH, Steece-Collier K (2003) The role of alpha-synuclein in Parkinson's disease: insights from animal models. Nat Rev Neurosci 4:727-738

47. McNaught KS, Belizaire R, Jenner P, Olanow CW, Isacson O (2002) Selective loss of 20S proteasome alpha-subunits in the substantia nigra pars compacta in Parkinson's disease. Neurosci Lett 326:155-158

48. Sullivan PG, Dragicevic NB, Deng JH, Bai Y, Dimayuga E, Ding Q, Chen Q, Bruce-Keller AJ, Keller JN (2004) Proteasome inhibition alters neural mitochondrial homeostasis and mitochondria turnover. J Biol Chem 279:20699-20707

49. Nakaso K, Yoshimoto Y, Yano H, Takeshima T, Nakashima K (2004) p53-mediated mitochondrial dysfunction by proteasome inhibition in dopaminergic SH-SY5Y cells. Neurosci Lett 354:213-216

50. Kikuchi S, Shinpo K, Tsuji S, Takeuchi M, Yamagishi S, Makita Z, Niino M, Yabe I, Tashiro K (2003) Effect of proteasome inhibitor on cultured mesencephalic dopaminergic neurons. Brain Res 964:228-236

51. Hoglinger GU, Carrard G, Michel PP, Medja F, Lombes A, Ruberg M, Friguet B, Hirsch EC (2003) Dysfunction of mitochondrial complex I and the proteasome: interactions between two biochemical deficits in a cellular model of Parkinson's disease. J Neurochem $86: 1297-1307$

52. Tanaka Y, Engelender S, Igarashi S, Rao RK, Wanner T, Tanzi RE, Sawa A, Dawson L, Dawson TM, Ross CA (2001) Inducible expression of mutant alpha-synuclein decreases proteasome activity and increases sensitivity to mitochondria-dependent apoptosis. Hum Mol Genet 10:919-926

53. Manila PM, Rinne JO, Helenius H, Dickson DW, Roytta M (2000) Alpha-synuclein-immunoreactive cortical Lewy bodies are associated with cognitive impairment in Parkinson's disease. Acta Neuropathologica 100:285-290 